\title{
Awareness about oral health in diabetic patients- A cross-sectional study
}

\author{
Maya S Indurkar'1, Krutika Thakur ${ }^{2, *}$, Sanjiv Indurkar ${ }^{3}$ \\ ${ }^{1}$ Professor and HOD, ${ }^{2}$ PG Student, ${ }^{3}$ Diabetolgist, ${ }^{\mathbf{1}, 2}$ Dept of Periodontology, ${ }^{3}$ Dept of Diabetology, Goverment Dental College and \\ Hospital Aurangabad, Maharashtra, India
}

*Corresponding Author:

Email: krutika2455@gmail.com

\begin{abstract}
Diabetes Mellitus is a chronic disease affecting all age groups and is one of the main causes for mortality and morbidity. Association between diabetes and periodontitis has been proven in the past and can be responsible for progression and complications of periodontitis.

Materials and Methods: 300 diabetic patients were given a questionnaire. Data was collected regarding the awareness among about the correlation between diabetes and periodontitis. Data was statistically analyzed using chi square test.

Results: On analysis, there was evident lack of awareness about the relation between diabetes and periodontitis.

Conclusion: There is a need to create awareness about how diabetes not only affects the general health but also affects the oral health of diabetic patients. They should also be motivated to maintain better oral hygiene and quit habits such as tobacco chewing, smoking, alcohol etc.
\end{abstract}

Keywords: Diabetes, Periodontitis, Gingivitis, Oral health, Gum health.

\section{Introduction}

Oral health is an integral part of systemic health and it plays a significant role in the maintenance of optimum general health. Several factors affect oral health which include various metabolic disorders one of which is diabetes mellitus. Diabetes is a clinically and genetically heterogeneous group of metabolic disorders caused by abnormally high levels of glucose in the blood. This hyperglycemia results from either a deficiency of insulin secretion caused by pancreatic $\beta$-cell dysfunction or resistance to the action of insulin in liver and muscles or both. ${ }^{1}$ The countries with the largest number of diabetics are India, China and the US with India beginning to be regarded as the diabetic capital of the world. ${ }^{2}$

Several studies ${ }^{3-7}$ have indicated deficiencies in awareness about oral health in patients with diabetes. Additionally, most of these studies ${ }^{3,4,6}$ showed that a very low number of patients diagnosed with diabetes visit the dentist regularly for periodontal checkups, and many patients were unaware of the effect of diabetes on oral health. Allen et al. $^{8}$ reported that awareness of periodontal diseases among diabetes patients is very low compared with their reported knowledge of increased risks for heart disease, eye disease, kidney disease and circulatory problems.

Patients are usually aware about the 5 complications of diabetes such as retinopathy, neuropathy, nephropathy, atherosclerosis and impaired wound healing but are unaware of Periodontitis as the $6^{\text {th }}$ complication of diabetes mellitus. ${ }^{9}$ They are also not aware about other oral manifestations found in uncontrolled diabetic patients such as : dry mouth, halitosis, oral candidiasis, pus discharge, gingivitis, periodontitis, xerostomia, dental caries, tooth loss, burning mouth syndrome, angular chellitis, median rhomboid glossitis, benign migratory glossitis, denture stomatitis, implant failure and oro- antral fistula.

\section{Objectives}

The present study was a descriptive cross- sectional study and its objective was to evaluate the presence of awareness in diabetic patients regarding the following:

1. Diabetes mellitus and its relation with periodontitis.

2. Diabetes mellitus and its relation with other oral manifestations.

3. Teeth cleansing methods, frequency and the oral hygiene status in diabetic patients.

4. Habits and various mucosal/oral diseases in diabetic patients.

\section{Materials and Methods}

The present study was conducted at Indurkar's Diabetic care center Aurangabad, Maharashtra, India. 300 diabetic patients were given a questionnaire printed in English, Hindi and local language (Marathi) and data was collected to know how aware the patients are about the correlation between diabetes mellitus and periodontitis and other oral manifestations. Practise of teeth cleansing method and different habits which are contributing to different oral diseases. The patients were selected by the following inclusion criteria:

1. Patients diagnosed with type- 2 diabetes mellitus.

2. Patients under treatment for diabetes mellitus.

3. Willingness to participate in the study.

\section{Results and Discussion}

Data was collected and comparison of frequencies across the categories with the outcome has been done using chi square test. For all the statistical tests, $p<0.05$ was considered to be statistically significant, keeping $\alpha$ 
error at $5 \%$ and $\beta$ error at $20 \%$, thus giving a power to the study as $80 \%$.

Out of 300 patients $212(70 \%)$ were male and 88 $(30 \%)$ were females. The age range of patients was $20 \mathrm{yrs}$ to $80 y$ rs with a mean age of $52.94 \pm 11.674$.

Age of detection and duration of diabetes mellitus

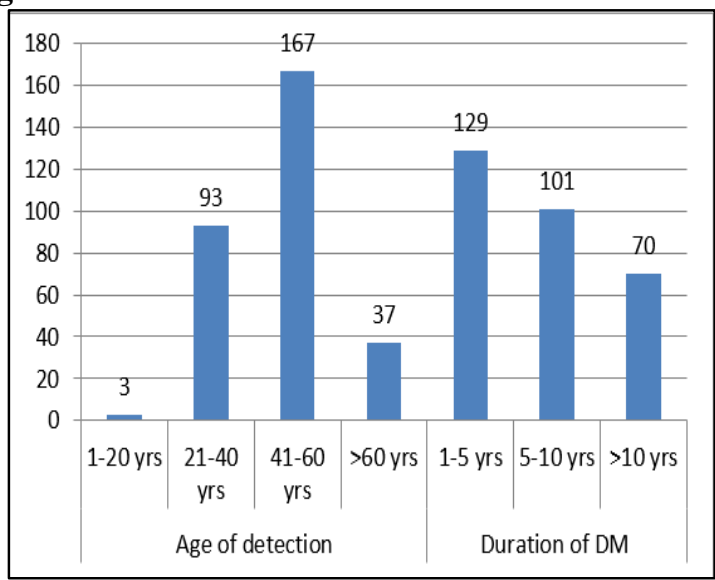

Fig. 1: Chart showing age of detection of DM and the duration of DM

Maximum number of patients i.e 167 patients $(55.6 \%)$ were detected with diabetes mellitus in the age group of 41 - 60 years and 93 patients $(31 \%)$ in the 12\%) were detected with diabetes above 60 years. According to the statistical analysis, there was a statistically significant correlation $(\mathrm{P}<0.05)$ between age of detection of DM in the age group 41- 60 years $(55.6 \%)$ and oral complaints of diabetic patients.

The duration of DM was 1 - 5 years in $129(43 \%)$ of the patients, $5-10$ years in 101 patients $(33.6 \%)$ and more than 10 years in 70 patients $(23.3 \%)$. The duration of DM was statistically correlated $(\mathrm{P}<0.05)$ with gingivitis and periodontitis since the $\mathrm{p}$ value was 0.04 .

Oral Hygiene Methods Habits and Diabetic Patients

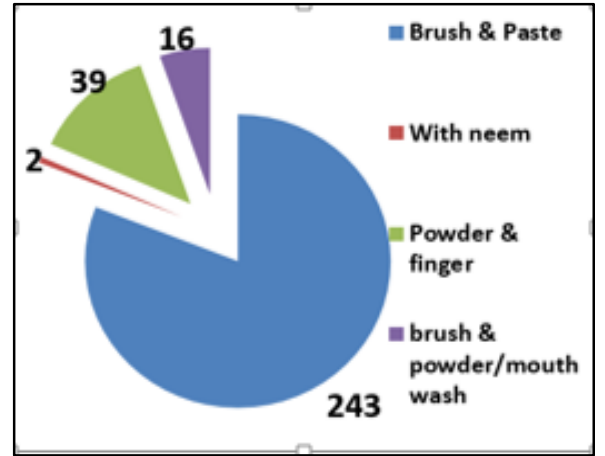

Fig 2: Showing oral hygiene methods in diabetic patients

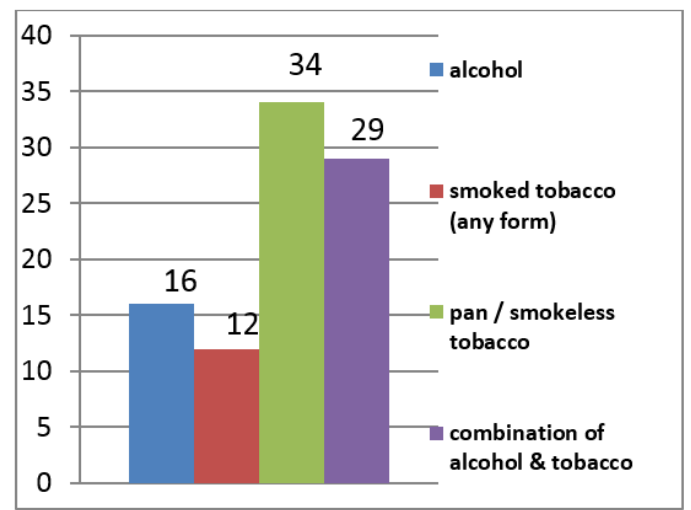

Fig. 3: Showing various habits in diabetic patients

Out of 300 diabetic patients 243 patients (81\%) used toothbrush and toothpaste for oral hygiene maintenance and the rest (19\%) either used powder, finger or mouth wash to maintain oral hygiene. Majority of the patients brushed once daily.

According to the figure above, out of 300 diabetic patients 91 patients $(30.3 \%)$ had various habits out of which 34 patients $(37 \%)$ had the habit of pan chewing or smokeless tobacco, 16 patients $(17.5 \%)$ had the habit of alcohol, 12 patients $(13.1 \%)$ had the habit of smoked tobacco (in any form) and 29 patients $(31.8 \%$ ) had a combined habit of alcohol and tobacco. The patients who had a habit of combination of alcohol and tobacco showed a greater statistically significant $(\mathrm{p}<0.05)$ correlation with periodontits in diabetic patients. Holbrook et al examined 524 adult diabetic patients and found that alcohol intake was a risk factor for development of diabetes in men. ${ }^{10}$ Diabetic patients should be motivated to control their habits such as tobacco, alcohol etc. as it not only affects their oral health but also affects the general health of the patient. 


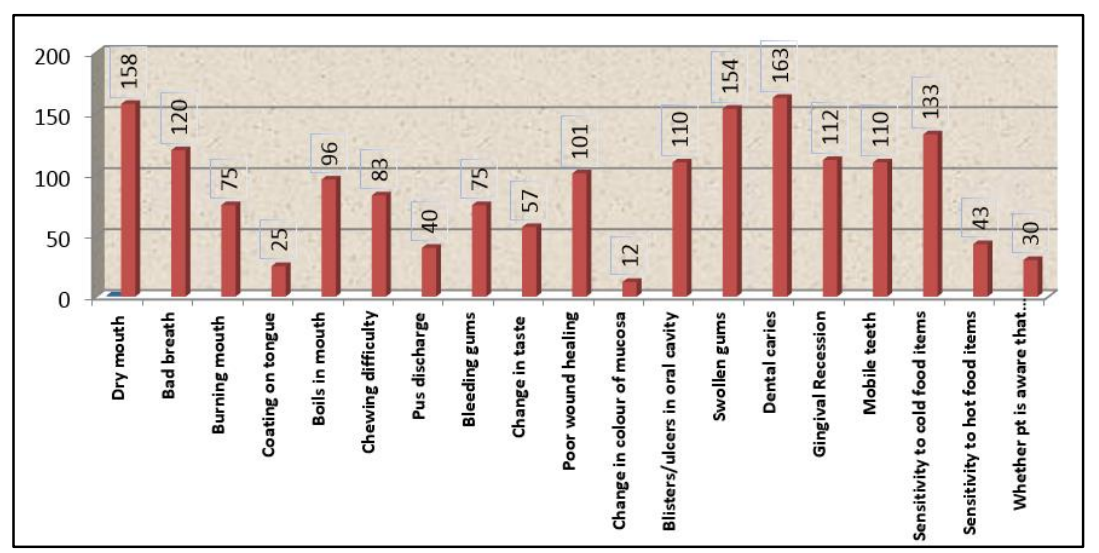

Fig. 4: Showing the various oral complaints of diabetic patients and the awareness about the correlation between DM and periodontitis

According to the above table, out of 300 diabetic patients, only $30(10 \%)$ were aware about the correlation between diabetes mellitus and periodontitis and other oral manifestations.

A cross-sectional survey was done in randomly selected general practitioners practicing in Kuwait by Areef K. et al. regarding the knowledge about association between diabetes mellitus and periodontal diseases. Out of 510 general practitioners (232 physicians and 278 dentists) who participated in the study $50 \%$ believed that patients with diabetes were more susceptible to tooth loss because of periodontal diseases than were individuals without diabetes. ${ }^{11}$

In the present study, among all the oral complaints, the following were reported more commonly.

Dry mouth and bad breath -158 patients $(52.6 \%)$ had acomplain of dryness of mouth and 120 patients (40\%) complained of bad breath. Xerostomia has been linked with dysfunction of the parenchyma of the major salivary glands and compensatory hyperplasia of the glandular parenchyma following a declining plasma insulin concentration. The constant dryness of the mouth reduced flushing action of saliva, high levels of ketones in the blood and high blood glucose level increases bacterial growth and build up of plaque in mouth which causes bad breath in diabetic patients. Chavez et al. found trends toward decreased salivary flow rates as $\mathrm{HbA1c}$ values increased. ${ }^{12} \mathrm{Kao} \mathrm{CH}$ et al. conducted a study which detected impaired salivary uptake and excretion by salivary scintigraphy in adults with type 2 diabetes ${ }^{13}$ Sandberg et al. (2000) found a significantly higher degree of xerostomia in type 2 diabetes mellitus. ${ }^{14}$

Dental caries-163 (54.3\%) Wegener ${ }^{15}$ in a study of 700 diabetic children, noted that immediately after onset of the disease, these children evidenced a higher caries incidence than controls. Decreased salivary secretion, increase of carbohydrate in the parotid gland saliva, increased glucose levels in GCF, growth of oral yeasts, increased counts of Streptococcus mutans and Streptococcus lactobacilli are some of the factors implicated to be responsible to predispose diabetics to higher incidence of dental caries. 163 i.e $54.3 \%$ patients had the complaint of dental caries and 154 i.e 51.3\% complained of dry mouth. This suggested that there could possibly be a correlation between xerostomia and occurrence of dental caries in diabetic patients. According to the study on dental caries prevalence among type 2 diabetic and non- diabetic adults attending hospital by Ekta A. et al. ${ }^{16}$ showed prevalence of dental caries of $73.33 \%$ in diabetics group and $33.33 \%$ among non- diabetic group.

Swollen gums 154 patients (51.3\%) bleeding gums75 patients (25\%) Recession 112 patients (37.3\%) Mobile teeth 110 patients (36.6\%) Poor wound healing101patients (33.6\%) and Sensitivity to cold food items $133(44.3 \%)$ All these complaints are suggestive of greater susceptibility of diabetic patients to gingivitis and periodontitis. Numerous contributing factors which are responsible for increased susceptibility of diabetic patients to periodontitis are compromised polymorphonuclear leukocyte function due to impaired neutrophil adherence, chemotaxis and phagocytosis. This prevents destruction of bacteria in the periodontal pocket and markedly enhances periodontal destruction. Abnormalities of collagen metabolism, impaired proliferation of osteoblasts, and weakened mechanical properties of newly formed bone have been documented in hyperglycemic patients. ${ }^{17,18}$ Patients with uncontrolled diabetes show exaggerated response to local factors such as plaque and calculus leading to the sequelae of gingivitis, periodontitis and alveolar bone loss. Graves and colleagues. ${ }^{19}$ reviewed the pathogenesis of periodontal disease in patients with diabetes and concluded that, in addition to the robust inflammatory response, enhanced apoptosis (the sequence of programmed events leading to cell death) may contribute to periodontitis as a complication of diabetes. This apoptosis is due to Advanced glycation end products in diabetic patients which lead to production of interleukin1 and tumor necrosis factor enhance the vulnerability to tissue destruction. ${ }^{17,20}$ Taylor $^{21}$ and Taylor and Borgnakke ${ }^{22}$ summarized the clinical studies in which 
periodontitis as a complication of diabetes mellitus was examined.

Boils-96 patients (32\%) ulcers and blisters -110 patients $(36.6 \%)$ and abcess/ pus discharge -40 patients (13.3\%) These were among the other complaints of diabetic patients which may be due to slower/delayed healing time microangiopathy or a defective function of the polymorphonuclear leukocytes. Altered or delayed wound healing may also play a role in traumatic ulcers. Guggenheimer and colleagues ${ }^{23}$ recently reported that people with DM have a higher prevalence of oral traumatic ulcers and irritation fibromas than do nondiabetic control subjects.

\section{Conclusion}

1. In the present survey $90 \%$ diabetic patients were not aware that they are more prone to diseases of gums, teeth, tongue and other mucosal diseases. Since the awareness among diabetic patients was limited and depicted poor knowledge and attitude towards oral health, creating awareness about the importance of maintaining good oral health is necessary.

2. Maximum diabetic patients i.e $243(81 \%)$ brushed only once daily. As the glucose levels are higher in saliva and crevicular fluids of diabetic patients which encourages bacterial growth and plaque accumulation patients should be motivated to brush twice daily to maintain better oral health.

3. Correlation of habits such as alcohol, smoking and tobacco with periodontitis in diabetic patients revealed significant association between them. Hence, patients should be informed about the illeffects of such habits on oral health and motivated to discontinue these habits.

4. The chances of the oral complications are minimized if the disease is well-controlled. A regular visit to the dentist \& physician is very important in diabetic patients for timely prevention and management of oral complications hence medical and dental practitioners should work in collaboration \& patient education should be considered an essential element.

\section{References}

1. Mealey BL, Ocampo GL. Diabetes mellitus and periodontal disease. Periodontol 2000-2007;44:127-53

2. Sharma A, Tiwari A. Diabetes mellitus and Dental Disease-a review. JIDA 2002;73:116-21.

3. Al Habashneh R, Khader Y, Hammad MM, et al. Knowledge and awareness about diabetes and periodontal health among Jordanians. J Diabetes Complications. 2010;24:409-14.

4. CDC. Dental visits among dentate adults with diabetes-United States, 1999 and 2004. MMWR Morb Mortal Wkly. Rep. 2005;54:1181-83.
5. Allen EM, Ziada HM, O'Halloran D, et al. Attitudes, awareness and oral health-related quality of life in patients with diabetes. J Oral Rehabil. 2008;35:218-23.

6. Jansson H, Lindholm E, Lindh C, et al. Type 2 diabetes and risk for periodontal disease: a role for dental health awareness. J Clin Periodontol. 2006;33:408-14.

7. Moore PA, Orchard T, Guggenheimer J, et al. Diabetes and oral health promotion: a survey of disease prevention behaviors. J Am Dent Assoc. 2000;131:1333-41

8. Allen EM, Ziada HM, O'Halloran D, et al. Attitudes, awareness and oral health-related quality of life in patients with diabetes. J Oral Rehabil. 2008;35:218-23.

9. Löe H. Periodontal disease: the sixth complication of diabetes mellitus. Diabetes Care. 1993;16(1):329-34.

10. Holbrook TL, Barrett-Conner E, Wingard DL. A prospective population- based study of alcohol use and non- insulin dependent diabetes mellitus. $A m j$ epidemiol. 1990:132:902-9

11. Areej K. Al-Khabbaz, Khalaf F. Al-Shammari, and Noha A. Al-Saleh Knowledge About the Association Between Periodontal Diseases and Diabetes Mellitus: Contrasting Dentists and Physicians. J Periodontol. 2011;82:83.

12. Chavez EM, Borrell LN, Taylor GW, et al. A longitudinal analysis of salivary flow in control subjects and older adults with type 2 diabetes. Oral Surg Oral Med Oral Pathol Oral Radiol Endod 2001;91:166-73.

13. Kao CH, Tsai SC, Sun SS. Scintigraphic evidence of poor salivary function in type II diabetes. Diabetes Care. 2001;24(5):952-53.

14. Sandberg GE, Sundberg HE, Fjellstrom CA, Wikblad KFl. Type 2 diabetes and oral health: a comparison between diabetic and non-diabetic subjects. Diabetes Res Clin Pract. 2000;50(1):27-34.

15. Wegener. H.,Dental caries in young diabeties. Caries Res. 1971;3:18-192.

16. Ekta A Malvania et al. Dental caries prevalence among type II diabetic and non- diabetic adults attending hospital. J Int Soc Prev Community Dent. 2016;6(3):S232-S36

17. Lalla RV, D'Ambrosio JA. Dental management considerations for the patient with diabetes mellitus. $J$ Am Dent Assoc. 2001;132:1425-32.

18. Ship JA. Diabetes and oral health: An overview. J Am Dent Assoc. 2003;134;4-10.

19. Graves DT, Liu R, Alikhani M, Al-Mashat H, Trackman PC. Diabetes-enhanced inflammation and apoptosis: impact on periodontal pathology. J Dent Res. 2006;85(1):15-21.

20. Mealey BL, Oates TW. Diabetes mellitus and periodontal disease. J Periodontol 2006;77:1289-303.

21. Taylor GW. Bidirectional interrelationships between diabetes and periodontal diseases: an epidemiologic perspective. Ann Periodontol. 2001;6(1):99-112.

22. Taylor GW, Borgnakke WS. Periodontal disease: associations with diabetes, glycemic control and complications. Oral Dis. 2008;14(3):191-203.

23. Guggenheimer J, Moore PA, Rossie, Myers D, Mongelluzzo MB, Block HM. Insulin-dependent diabetes mellitus and oral soft tissue pathologies. I. Prevalence and characteristics of non-candidal lesions. Oral Surg Oral Med Oral Pathol Oral Radiol Endod. 2000;89(5):563 -69. 